\title{
Evaluation and optimal scaling of distributed generation systems in a smart city
}

\author{
C. F. Calvillo, A. Sánchez \& J. Villar \\ Institute for Research in Technology (IIT), \\ Comillas Pontifical University, Spain
}

\begin{abstract}
Distributed generation (DG) represents an important resource to address relevant energy issues, such as reliability and sustainability, in the current and future smart cities. It is expected that distributed generation will gain considerable presence in the following years; however, the selection and sizing of the generation and storage systems is commonly done without an adequate level of detail. This simplified or approximated approach usually results in a suboptimal technology mix with an inadequate type of system and/or scale, which could compromise the economic feasibility of the DG project.

To tackle this problem, stakeholders should consider many factors, including geographical characteristics (sun, wind ...), energy costs, local regulation, and energetic demand patterns, apart from analysing different technologies. Considering as an example location the city of Madrid, Spain, this paper proposes a linear programming model to evaluate the most common distributed generation technologies, with and without storage systems and under different electricity pricing scenarios. As a result, not only the optimal sizing, but also the optimal operation scheduling of the aforementioned systems are found. Then, an economic feasibility analysis is developed, comparing the different technologies and defining the best option for a given scenario. Furthermore, this study helps to find important milestones, such as battery prices, that could make distributed generation more attractive.
\end{abstract}

Keywords: smart city, distributed generation, renewable sources, energy storage. 


\section{Introduction}

Energy is omnipresent in current cities. It is a prime element in modern urban life, as most of our everyday activities use some sort of energy. Unfortunately, with this increased dependency to energy, in addition to rapid urban growth rates, many energy sustainability, quality and reliability problems arise [1].

Distributed generation (DG) is a well-studied topic and it is considered a promising solution for the abovementioned issues; on the one hand, it facilitates the placing of generation sources closer to the loads, reducing losses and improving reliability, and on the other hand, it helps to integrate renewable energy into the system [2].

Renewable sources in DG schemes with and without energy storage are widely present in literature. Solar power is one of the most popular DG technologies and many examples of such systems can be found. For instance, in [3] a technical and economic analysis is carried out for solar photovoltaic (PV) and solar thermal technologies for heating, hot water and electricity production in a residential building. The drawback in this study is that their cost estimations are arbitrary and they do not review other applicable sources.

Wind power is also commonly found in DG applications. Yang et al. [4] presents a complete wind energy conversion system for a microgrid, including the required power electronics. The research mainly focuses on operation and control, disregarding energy storage as a complementary technology and economic analysis.

Cogeneration (heat and power) and polygeneration (heat, cooling and power) schemes are studied in [4], presenting a general model for energy production and emission performance; however, it doesn't includes economic aspects or comparison with other technologies.

Another interesting approach is the hybrid DG schemes, which contain more than one energy source. In [6], a feasibility analysis of geothermal heat pumps and other cogeneration technologies for buildings is presented. However, the proposed study lack detail in relevant aspects such as demand profiles, system costs and energy production. Finally, references [7] and [8] perform a technical and economic feasibility analysis of a wind-photovoltaic system with storage. Unlike most of the previous works, these studies do include an appropriate level of detail; however, they do not consider thermal energy, and lack a comparison with other technologies.

The main problem in the previous systems is that they mostly focus on operation, while overlooking the adequate selection and scaling of the systems. If relevant location aspects are not considered, and/or if the system is not optimally scaled given the application; then, accurate economic feasibility analyses cannot be performed, and might compromise the success of such DG projects. This paper address the aforementioned problem, and follows the work presented in [9], by proposing several linear programing problems to find the optimal scaling and operation of different generation technologies and storage systems under 9 different electricity pricing scenarios. The resulting economic analysis defines the best technology mix for every scenario, assisting investors in finding the 
most profitable system for the selected application while improving the reliability and sustainability of the energy supply.

\section{Generation models}

Many available technologies can be successfully implemented in a smart city. A comprehensive revision of such technologies is presented in [9], remarking their advantages and disadvantages, typical applications and costs.

Solar photovoltaic systems can be described with the following equation:

$$
\text { energy }_{P V}(t)=\frac{\text { power }_{\text {peak }}}{G} * D N I(t) * T *\left(1-\frac{\text { losses } \%}{100}\right)
$$

where: DNI stands for Direct Normal Irradiance $\left(\mathrm{W} / \mathrm{m}^{2}\right)$, which is the energy provided by the sun at period $t$ in the specified location. The peak power $(\mathrm{kW})$ is provided by the fabricant. $T$ is the duration of the period (for this research, $T$ will always be 1hour). Lastly, $G$ is the global irradiation received on a horizontal plane $=1000 \mathrm{~W} / \mathrm{m}^{2}$.

Continuing with solar power, the thermal collector (TC) is modelled with eqn (2). This equation differs from the previous one in that the peak power is a function of the difference between the fluid inlet and ambient temperatures, as described in eqn (3). The peak power function is normally provided by the fabricant.

$$
\begin{gathered}
\text { energy }_{T C}(t)=\frac{\text { power }_{\text {peak }}(p)}{G} * D N I(t) * T *\left(1-\frac{\text { losses } \%}{100}\right) \\
p=T_{\text {inlet }}-T_{\text {ambient }}
\end{gathered}
$$

Wind power can be calculated as a function of the wind speed $V_{\text {wind }}(\mathrm{m} / \mathrm{s})$ with eqn (4), where the power function is given by the wind turbine fabricant.

$$
\text { energy }_{W T}(t)=\text { power }_{W T}\left(V_{\text {wind }}(t)\right) * T *\left(1-\frac{\text { losses } \%}{100}\right)
$$

The thermal production of a geothermal heat pump (GHP) can be calculated with eqn (5). The Coefficient of Performance (CoP) is used to measure the thermal output given the electric energy input $E(t)$.

$$
\text { energy }_{\text {GeoHP }}(t)=\mathrm{CoP}^{*} \mathrm{E}(t) * T *\left(1-\frac{\text { losses } \%}{100}\right)
$$

Cogeneration is modelled in a generic CHP approach, presented in eqn (6), where $P(t)$ and $Q(t)$ stand for the electric and thermal production at period $t$.

$$
\text { energy }_{C H P}(t)=P(t)+Q(t)
$$

Eqns (7) and (8) are used to compute the two energy vectors. On the one hand, the electric and thermal efficiency ratios: $\eta_{e}$ and $\eta_{t}$ are provided by the CHP unit fabricant. On the other hand, $F(t)(\mathrm{kW})$ is the power provided by the input fuel.

$$
\begin{gathered}
P(t)=\eta_{e} * F(t) * T *\left(1-\frac{\text { losses }_{e} \%}{100}\right) \\
Q(t)=\eta_{t} * F(t) * T *\left(1-\frac{\text { losses }_{t} \%}{100}\right)
\end{gathered}
$$

Finally, geothermal cogeneration can be implemented for medium to high scale applications. This plant can be modelled as a steam turbine CHP, as in eqn (6). 
Steam is passed through a turbine to produce electricity with efficiency $\eta_{e}$, and the resting thermal energy in the exhaust steam is used for heating purposes. Eqns (9) an (10) describe the electric and thermal production, and eqn (11) presents the relation between the energy in the input and output steam.

$$
\begin{array}{r}
P(t)=\eta_{e} * e_{\text {inlet }} * T *\left(1-\frac{\text { losses }_{e} \%}{100}\right) \\
Q(t)=e_{\text {exhaust }} * T *\left(1-\frac{\text { losses }_{t} \%}{100}\right) \\
e_{\text {exhaust }}=\left(1-\eta_{e}\right) * e_{\text {inlet }}
\end{array}
$$

\section{Linear programing problem}

This section describes the optimization models created to find the optimal scaling and operation of the reviewed generation and storage technologies. It is important to remark that the generation and storage systems in this study are treated and compared independently; therefore, a different liner programming model was created for every generator and every scenario.

\subsection{Nomenclature}

The general nomenclature of the generation and storage models is presented next. As mentioned above, different models were created; thus, not all variables are used in all models.

\begin{tabular}{|c|c|}
\hline lifespan & Expected lifespan of the system (years) \\
\hline demandCurve $_{m, h}$ & Normalized electric demand curve (\%) (Figure 1a) \\
\hline demElecMens ${ }_{m}$ & Normalized electric demand evolution (\%) (Figure 1b) \\
\hline demandElecAnnual & Total annual electric demand $(\mathrm{kWh})$ \\
\hline demandElec $_{m, h}$ & Electric demand curve for 12 representative days (kW) \\
\hline demandThermAnnual & Total annual thermal demand $(\mathrm{kWh})$ \\
\hline emandTherm $_{m}$ & Total thermal demand for 12 representative days $(\mathrm{kW})$ \\
\hline hourlyProfile $_{m, h}$ & Normalized electric hourly prices (Figure 2) \\
\hline monthProfile $_{m}$ & Normalized electric prices evolution in a year \\
\hline hourlyPrice $_{m, h}$ & Absolute electric hourly prices (USD/kWh) \\
\hline costEbase & Electric energy price at year 0 (USD/kWh) \\
\hline costTbase & Thermal energy price at year 0 (USD/kWh) \\
\hline costE $E_{y}$ & Electric energy price at year $y$ (USD/kWh) \\
\hline $\operatorname{cost} T_{y}$ & Thermal energy price at year $y$ (USD/kWh) \\
\hline$D N I_{m, h}$ & Direct normal irradiance at month $m$, hour $h(\mathrm{~W})$ \\
\hline ssesE & Total electric losses (\%) \\
\hline sesT & Total thermal losses (\%) \\
\hline costInst & Cost per installed Watt of technology $X$ (USD/W) \\
\hline
\end{tabular}

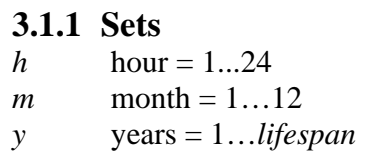

\subsubsection{Parameters}




\begin{tabular}{|c|c|}
\hline OyMfix & $\begin{array}{l}\text { Fixed annual Operation and Maintenance costs per installed Watt } \\
\text { for technology } X(\mathrm{USD} / \mathrm{W})\end{array}$ \\
\hline OyMvar & $\begin{array}{l}\text { Variable annual operation and Maintenance costs per produced } \\
\text { kWh for technology } X(\mathrm{US} \Phi / \mathrm{kWh})\end{array}$ \\
\hline effElec & Electric energy production efficiency ratio (\%) \\
\hline effTerm & Thermal energy production efficiency ratio (\%) \\
\hline daysInMonth $_{m}$ & Number of days in month $m$ \\
\hline discountRate & Standard discount rate for project comparison (\%) \\
\hline priceIncRate & Annual increment rate in energy prices (\%) \\
\hline effBat & Battery charge/discharge efficiency ratio (\%) \\
\hline $\begin{array}{l}\text { 3.1.3 Variables } \\
\text { powerInst }\end{array}$ & Installed capacity of technology $X(\mathrm{~kW})$ \\
\hline boughtEnergy $E_{m, h}$ & $\begin{array}{l}\text { Electricity bought from the grid to meet the demand at month } m \text {, } \\
\text { hour } h(\mathrm{kWh})\end{array}$ \\
\hline boughtEnergy $T_{m}$ & $\begin{array}{l}\text { Thermal energy bought from the grid to meet the daily demand } \\
\text { in month } m(\mathrm{kWh})\end{array}$ \\
\hline prodElec $_{m, h}$ & $\begin{array}{l}\text { Electric demand minus electric production at month } m \text {, hour } h \\
(\mathrm{kWh})\end{array}$ \\
\hline prodTherm $_{m}$ & $\begin{array}{l}\text { Daily thermal demand minus daily thermal production at month } m \\
(\mathrm{kWh})\end{array}$ \\
\hline fuel $_{m, h}$ & Input fuel at month $m$, hour $h(\mathrm{kWh})$ \\
\hline batCapacity & Installed capacity of the battery system (kWh) \\
\hline$S O C_{m, h}$ & Battery State-of-Charge at month $m$, hour $h(\mathrm{kWh})$ \\
\hline dischargeBat $_{m, h}$ & Energy discharged from battery at month $m$, hour $h(\mathrm{kWh})$ \\
\hline chargeBat $_{m, h}$ & Energy charged to the battery at month $m$, hour $h(\mathrm{kWh})$ \\
\hline boughtEnergyCh ${ }_{m, h}$ & $\begin{array}{l}\text { Energy bought from the grid to charge the batteries at month } m \text {, } \\
\text { hour } h(\mathrm{kWh})\end{array}$ \\
\hline
\end{tabular}

\subsection{Mathematical formulation}

This section presents the detailed mathematical formulation of the linear programing models used to find the optimal scaling and operation of the DG systems.

\subsubsection{Objective function}

The general objective function is to maximize the benefits of the reviewed configurations, considering the total lifetime of the project and battery replacement every 8 years.

$$
\begin{aligned}
& \max \left\{\sum_{y}\left[\text { Cost }_{y} * \sum_{m}\left[\text { daysInMonth }_{m} * \sum_{h}\left[\text { hourlyPrice }_{m, h} * \text { generation }_{m, h}\right]\right]\right]\right. \\
&- \text { CostInst } * \text { powerInst }- \text { OyMfix } * \text { powerInst } * \text { lifespan } \\
&\left.- \text { CostBat }^{*} \text { capacityBat }^{\text {Lifespan }} / 8\right\} \\
& \text { generation }_{m, h}=\text { demandElec }_{m, h}-\text { boughtEnergy }_{m, h}
\end{aligned}
$$

\subsubsection{Constraints}

Note that all indexed variables apply for all $m$ in month and for all $h$ in hour; unless explicitly stated differently. 


\section{State-of-charge constraints}

$$
\begin{gathered}
\operatorname{SOC}[1][0]=0 \\
S O C[m][1]=S O C[m][24] \quad \forall m \in[2, \ldots, 11] \\
S O C[m][0]=S O C[m-1][24] \quad \forall m \in[2, \ldots, 12] \\
\text { batCapacity } \geq S O C[m][h] \geq 0 \\
\operatorname{abs}(\operatorname{SOC}[m][h]-S O C[m][h-1]) \leq 1 \\
S O C[m][h]=S O C[m][h-1]-\text { dischargeBat }[m][h]+\text { chargeBat }[m][h]
\end{gathered}
$$

\section{Charge/discharge constraints}

$$
\begin{gathered}
\text { dischargeBat }[m][h] \geq 0 \\
\text { dischargeBat }[m][h] \leq S O C[m][h-1] \\
\operatorname{chargeBat}[m][h] \geq 0 \\
\text { chargeBat }[m][h] \leq \text { batCapacity }-\operatorname{SOC}[m][h-1]
\end{gathered}
$$

\section{Bought energy constraints}

$$
\begin{aligned}
& \text { boughtEnergy } E[m][h] \geq 0 \\
& \text { boughtEnergy } T[m][h] \geq 0 \\
& \text { boughtEnergyCh }[m][h] \geq 0
\end{aligned}
$$

\section{Energy production constraints}

In eqns (27) and (28), prodDG refers to the energy produced by the selected generation technology, corresponding to the equations described in Section 2. Thermal generation is considered greater than its actual production as it is compared with the cost of producing the same amount of energy with a conventional gas boiler at $80 \%$ efficiency.

$$
\begin{aligned}
& \operatorname{prodElec}[\mathrm{m}][\mathrm{h}]=\text { demandElec }[\mathrm{m}][\mathrm{h}]-\operatorname{prodDGelec}[\mathrm{m}][\mathrm{h}] \\
& \operatorname{prodTherm}[\mathrm{m}]=\text { demandTherm }[\mathrm{m}]-\operatorname{prodDGtherm}[\mathrm{m}] / 0.8
\end{aligned}
$$

Constraint (29) applies for the cogeneration cases, where the electric production cannot go above the nominal installed power.

$$
\text { prodDGelec }[m][h] \leq \text { powerInst }
$$

The following set of constraints refers to the difference between DG production and demand, due to the change in behaviour of the model according to the case.

prodElec $[m][h]>0 \Rightarrow$

boughtEnergyE $[m][h]=\operatorname{prodElec}[m][h]-\operatorname{dischargeBat}[m][h]+\operatorname{boughtEnergyCh}[m][h]$

chargeBat $[m][h] \leq$ effBat *boughtEnergyCh $[m][h]$

prodElec $[m][h] \leq 0 \Rightarrow$

$$
\begin{gathered}
\text { boughtEnergyE }[\mathrm{m}][h]=\text { boughtEnergyCh }[\mathrm{m}][h] \\
\text { chargeBat }[\mathrm{m}][h] \leq \operatorname{effBat} *(\text { abs }(\text { prodElec }[\mathrm{m}][h])+\text { boughtEnergyCh }[\mathrm{m}][h]) \\
\operatorname{prodTherm}[\mathrm{m}][h]>0 \Rightarrow \text { boughtEnergyT }[\mathrm{m}]=\operatorname{prodTherm}[\mathrm{m}] \\
\operatorname{prodTherm}[\mathrm{m}][h] \leq 0 \Rightarrow \text { boughtEnergyT }[\mathrm{m}]=0
\end{gathered}
$$




\section{Scenarios and case studies}

Two case studies were developed in this paper, considering the geographical characteristics of Madrid, Spain. Case A refers to a very small scale application, i.e. one household; while, Case B considers the medium scale in a district application, i.e. 100 houses. Table 1 shows the average domestic energy consumption per year in Spain, with the corresponding electrical and thermal shares [10].

Table 1: Average annual energy consumption per household in Spain (2011).

\begin{tabular}{lcccc}
\hline $\begin{array}{c}\text { Consumption per } \\
\text { Household }\end{array}$ & Electric (e) & $\begin{array}{c}\text { Thermal (t): } \\
\text { DHW/Heating } \\
\text { Energy }(\mathrm{kWh})\end{array}$ & $\begin{array}{c}\text { Thermal: } \\
\text { Others }\end{array}$ & Total \\
Percentage (\%) & 3698.13 & 6384.85 & 438 & 10.521 \\
\hline
\end{tabular}

a) Typical electric demand curves

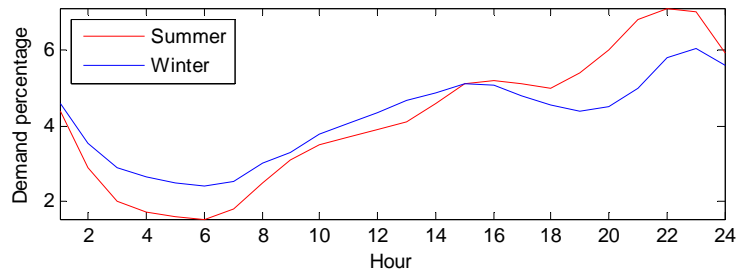

b) Annual demand evolution

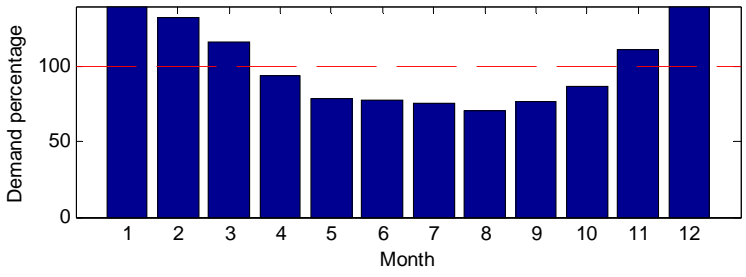

Figure 1: Demand curves and annual evolution for residential sector in Spain.

Additionally, electric demand curves are required to accurately calculate the optimal generation and storage capacity to be installed. With the data extracted from [11], Figure 1a presents the electric demand curves for a typical day in summer and winter, and Figure 1b show the evolution of the demand throughout a regular year.

\subsection{Electric energy and cogeneration based scenarios}

In this study, 9 electricity pricing scenarios were created to test the different generation technologies with and without storage under both study cases. The first scenario corresponds to the simplest configuration without storage and with static electricity price lacking hourly discrimination. The second and third 
scenarios have the same pricing but with energy storage; the difference between them is that the former uses the extra generation to charge the batteries, while the latter can also buy energy from the grid for charging. Scenarios 4, 5 and 6 have hourly price discrimination with three tariffs: peak, off-peak and valley. The difference among them is the existence of energy storage and the charging methods as in scenarios 2 and 3.

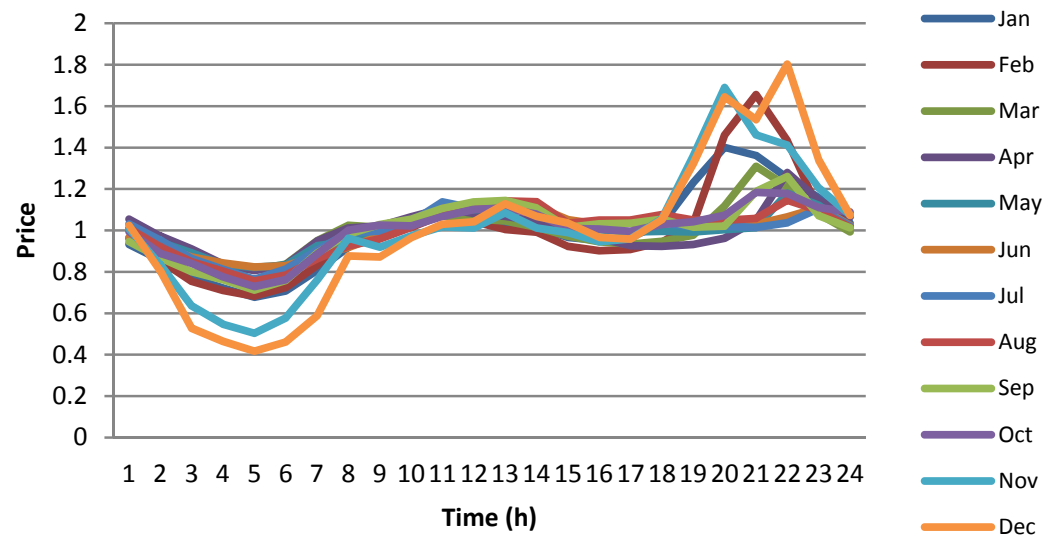

Figure 2: $\quad$ Average spot prices per month for the Spanish market in 2009.

Scenarios 7 to 9 consider a dynamic market pricing scheme as in the Spanish wholesale energy market. Currently, this pricing does not exist for small or medium consumers; however, it is expected that a similar approach will be available in the following years. In these scenarios, spot prices of the primary market were scaled to provide same average value as the actual electricity price, as shown in Figure 2. Finally, Table 2 summarizes the 9 presented scenarios.

Table 2: $\quad$ Pricing and storage scenarios.

\begin{tabular}{ccll}
\hline Scenario & Electric storage & Charging method & \multicolumn{1}{c}{ Pricing method } \\
1 & No & - & Static \\
2 & Yes & DG only & Static \\
3 & Yes & DG + grid & Static \\
4 & No & - & Peak, Off-peak, Valley \\
5 & Yes & DG only & Peak, Off-peak, Valley \\
6 & Yes & DG + grid & Peak, Off-peak, Valley \\
7 & Yes & - & Market spot price \\
8 & Yes & DG only & Market spot price \\
9 & Yes & DG + grid & Market spot price \\
\hline
\end{tabular}

\subsection{Thermal energy based scenarios}

As thermal energy (natural gas or other fossil fuel) is traded to the end-user in a more static fashion than electricity, a single scenario is considered for the thermal-only DG technologies. Furthermore, TC and GHP systems include water 
tanks as thermal storage; thus, dimensioning of these thermal schemes can be done in a straightforward manner.

\section{Economic analysis and results}

Apart from the optimal scaling and operation profiles of the reviewed systems, the linear programing problems also provide an economic analysis, where the value of the objective function is equivalent to the Net Present Value (NPV) of the project at the end of the expected lifespan, with a discount rate of $5 \%$. For the sake of comparison, the assumed lifespan of all projects is 20 years. Table 3 shows the generators data for the economic analysis, including data collected from [12-15]; while energy tariffs and time characteristics are summarized in Table 4, It is assumed an 8\% of energy price increment per year. Real battery system price is approximately 0.6 USD per installed Wh.

Table 3: $\quad$ Generators costs and expected energy losses.

\begin{tabular}{|c|c|c|c|c|c|c|c|}
\hline \multirow[t]{2}{*}{ Tech. } & \multicolumn{2}{|c|}{$\begin{array}{l}\text { Cost/W } \\
(\text { USD/W } \\
\text { p) }\end{array}$} & \multicolumn{2}{|c|}{$\begin{array}{l}\text { O\&M }_{\text {fix }} \\
\text { (USD/ kW) }\end{array}$} & \multicolumn{2}{|c|}{$\begin{array}{l}\text { O\&M }_{\text {var }} \\
\text { (US\$/ kWh) }\end{array}$} & \multirow[t]{2}{*}{$\begin{array}{l}\text { Losses } \\
(\%)\end{array}$} \\
\hline & $\boldsymbol{h}$ & $d$ & $\boldsymbol{h}$ & $d$ & $h$ & $d$ & \\
\hline PV & 4.1 & 2.6 & 63 & 45 & - & - & $24 \mathrm{e}$ \\
\hline WT & 5.0 & 3.4 & - & - & 3.5 & 2.8 & $20 \mathrm{e}$ \\
\hline $\mathrm{TC}$ & 2.7 & 2.0 & 50 & 35 & - & - & $15 t$ \\
\hline $\begin{array}{l}\text { Geo- } \\
\text { HP }\end{array}$ & 4.8 & 4.1 & 150 & 180 & - & - & $15 t$ \\
\hline CHP & $6.3^{1}$ & $2.0^{2}$ & 70 & 65 & 3.2 & 3.0 & $20 \mathrm{e} / 15 \mathrm{t}$ \\
\hline
\end{tabular}

${ }^{1}$ fuel cell; ${ }^{2}$ microturbine; h: household; d: district; e: electric; t: thermal.

Table 4: $\quad$ Peak, off-peak and valley time schedule and pricing.

\begin{tabular}{lccc}
\hline $\begin{array}{c}\text { Three tariffs } \\
\text { (electricity) }\end{array}$ & Peak & Off-Peak & Valley \\
\hline Winter & $18-22 \mathrm{~h}$ & $\begin{array}{c}8-18 \mathrm{~h} \text { and } \\
22-24 \mathrm{~h}\end{array}$ & $0-8 \mathrm{~h}$ \\
& & $\begin{array}{c}8-11 \mathrm{~h} \text { and } \\
15-24 \mathrm{~h}\end{array}$ & $0-8 \mathrm{~h}$ \\
Summer & $11-15 \mathrm{~h}$ & 0.0893 & 0.0639 \\
& & Natural gas & \\
Price (USD/kWh) & 0.2156 & 0.0641 & \\
\hline \hline Static tariff & Electricity & 0.1864 & \\
Price (USD/kWh) &
\end{tabular}

Solar production is calculated with hourly DNI data from [16]. On the contrary, a standard wind production profile for the selected location is difficult to formulate due to high variability of wind; therefore, the energetic production is approximated using average wind speed values, acquired from [17].

A first run of the optimization models was carried out and the results obtained showed that current battery prices are very high, so storage systems were not implemented in most cases. In order to examine the behaviour of storage in the proposed scenarios, different battery prices were tested, as shown in Figure 3. For this exercise, the battery price was established to 0.2 USD/Wh.

Results of the study for the electric energy based scenarios are summarised in Tables 5 to 7 . For the study case A (1 household), wind and solar power are 


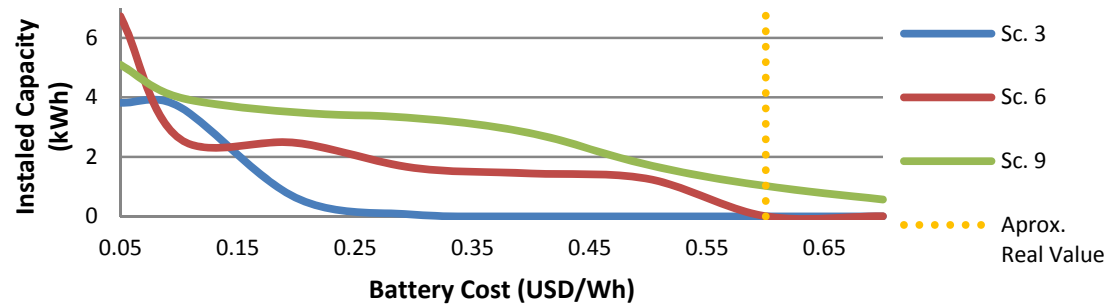

Figure 3: $\quad$ Battery price vs. installed capacity in the PV + storage system.

profitable, except for the "three price" scenarios. On the other hand, the available technology for small scale CHP is the fuel cell, which has a very high price, making it unfeasible under any scenario. In the study case B (1 district), solar and wind power are now producing benefits in every scenario; while the lower price of other CHP technologies, make it feasible in most scenarios; yet, less profitable than the PV and WT. it is important to remark that, storage systems are proportionally smaller than in the 1 household case.

Table 5: $\quad$ Installed power $(\mathrm{kW})$.

\begin{tabular}{lllllllllll}
\hline \multicolumn{2}{c}{ Tech. } & Sc.1 & Sc.2 & Sc.3 & Sc.4 & Sc.5 & Sc.6 & Sc.7 & Sc.8 & Sc.9 \\
PV & $h$ & 0.67 & 0.79 & 0.79 & 0 & 0 & 0 & 0.77 & 1.41 & 1.24 \\
& $d$ & 55.17 & 54.16 & 165.82 & 55.17 & 55.17 & 54.16 & 100.96 & 100.44 & 101.23 \\
WT & $h$ & 1.68 & 1.82 & 1.823 & 0 & 0 & 0 & 2.07 & 2.40 & 3.06 \\
& $d$ & 232.24 & 258.63 & 91.84 & 106.32 & 122.84 & 103.59 & 272.64 & 270.51 & 265.32 \\
CHP & $h$ & 0 & 0 & 0 & 0 & 0 & 0 & 0 & 0 & 0 \\
& $d$ & 24.32 & 24.32 & 24.32 & 0 & 0 & 0 & 44.22 & 44.51 & 50.29 \\
\hline
\end{tabular}

h: household; d: district.

Table 6: $\quad$ Electric storage capacity $(\mathrm{kWh})$.

\begin{tabular}{clllllll}
\hline \multicolumn{2}{c}{ Tech. } & Sc.2 & Sc.3 & Sc.5 & Sc.6 & Sc.8 & Sc.9 \\
PV & $h$ & 0.61 & 0.61 & 0 & 2.46 & 2.66 & 3.49 \\
& $d$ & 266.14 & 373.99 & 0 & 4 & 6.64 & 11 \\
WT & $h$ & 0.47 & 0.47 & 0 & 2.07 & 0.94 & 1.36 \\
& $d$ & 116.29 & 0 & 4 & 4 & 11 & 10.69 \\
CHP & $h$ & 0 & 0 & 0 & 2.07 & 0 & 3 \\
& $d$ & 0 & 0 & 0 & 4 & 1.68 & 14.21 \\
\hline
\end{tabular}

h: household; d: district.

Table 7: $\quad$ Objective function or Net Present Value (kUSD).

\begin{tabular}{lllllllllll}
\hline \multicolumn{2}{c}{ Tech. } & Sc.1 & Sc.2 & Sc.3 & Sc.4 & Sc.5 & Sc.6 & Sc.7 & Sc.8 & Sc.9 \\
PV & $h$ & 1.48 & 1.53 & 1.53 & 0 & 0 & 1.42 & 2.44 & 3.21 & 5.32 \\
& $d$ & 263.32 & 333.83 & 355.81 & 67.04 & 67.04 & 70.40 & 373.84 & 378.73 & 383.15 \\
WT & $h$ & 1.58 & 1.85 & 1.86 & 0 & 0 & 1.16 & 3.77 & 4.69 & 3.48 \\
& $d$ & 477.34 & 543.50 & 264.55 & 12.75 & 14.24 & 16.07 & 762.58 & 773.81 & 759.68 \\
CHP & $h$ & 0 & 0 & 0 & 0 & 0 & 1.09 & 0 & 0 & 0.91 \\
& $d$ & 14.67 & 14.67 & 14.67 & 0 & 0 & 3.70 & 57.42 & 57.58 & 52.25 \\
\hline
\end{tabular}

h: household; d: district. 
An example of the operation schedule obtained for a PV system in a winter day under the scenario 9 is presented in Figure 4. In this example, energy is bought to charge batteries when the energy price is low (early morning). In the middle of the day, PV production starts and, combined with some energy of the batteries, meets the demand. Finally, in peak hours, all the stored energy is used to avoid buying electricity at high prices.

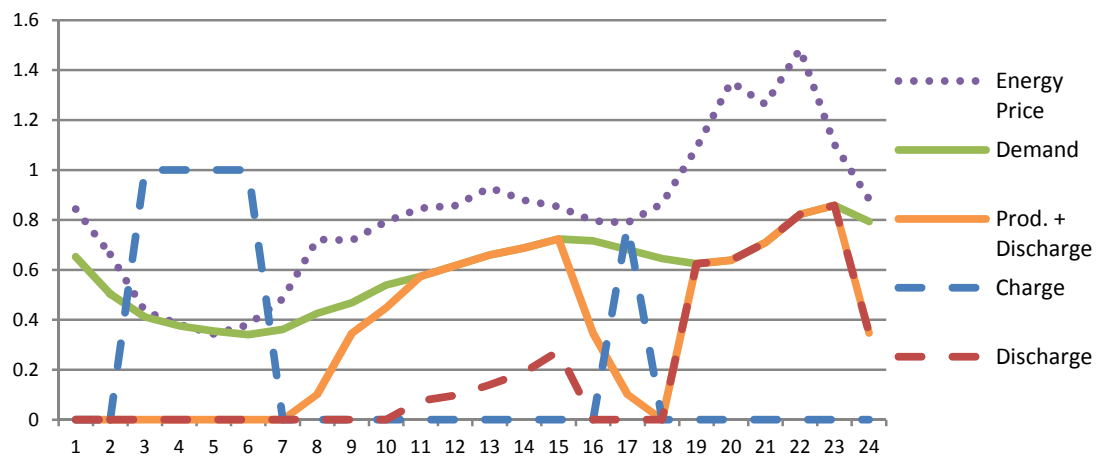

Figure 4: Operation behaviour for a PV system in a typical day of January.

Focusing on the thermal energy based scenario, Table 8 presents the required installed power and benefit achieved per system. Even though both systems are profitable, the flexibility of the geothermal heat pump production gives an advantage in the final result; however, the simplicity of the TC system makes it an attractive alternative, especially in the small scale application.

Table 8: Thermal energy scenario results.

\begin{tabular}{lcccc}
\hline Technology & \multicolumn{2}{c}{ Inst. Power (kW) } & \multicolumn{2}{c}{ Obj. Function (kUSD) } \\
& $h$ & $d$ & $h$ & $d$ \\
TC & 1.52 & 157.97 & 0.37 & 83.33 \\
Geo-HP & 0.25 & 19.89 & 1.65 & 130.50 \\
\hline
\end{tabular}

\section{Conclusions}

This paper models common distributed generation and storage systems for the smart city. Linear programming problems were proposed, considering solar, wind, and energetic demand characteristics in Madrid, Spain. As a result, optimal scaling and operation scheduling was found, in addition to economic feasibility analyses for different size of applications and several price scenarios. In this study, it was established that electric storage systems, can be very attractive in DG systems by taking advantage of fluctuating energy prices and adding flexibility in to the system; however, battery prices are still too high and they should go down considerably (about three times) in order to be profitable with current electricity tariffs. Regarding to generation technologies, despite the fact 
that some of them are already profitable in the small scale, others like the CHP fuel cell are completely out priced; nonetheless, many technologies can be competitive in the medium to high scale.

In future work, energy transactions between agents and energy markets will be included for a complete microgrid model.

\section{References}

[1] Cossent, Rafael, Tomás Gómez, and Luis Olmos. "Large-scale Integration of Renewable and Distributed Generation of Electricity in Spain: Current Situation and Future Needs." Energy Policy 39, no. 12 (December 2011): 8078-8087.

[2] Cossent, Rafael, Tomás Gómez, and Pablo Frías. “Towards a Future with Large Penetration of Distributed Generation: Is the Current Regulation of Electricity Distribution Ready? Regulatory Recommendations under a European Perspective.” Energy Policy 37, no. 3 (March 2009): 1145-1155.

[3] Adhikari, R.S., M. Buzzetti, and S. Magelli. "Solar Photovoltaic and Thermal Systems for Electricity Generation, Space Heating and Domestic Hot Water in a Residential Building." In 2011 International Conference on Clean Electrical Power (ICCEP), 461-465, 2011.

[4] Yang, Wang, Wu Zai-jun, and Li Zhengming. "Research on Wind Energy Distributed Generation in Microgrid." In 2010 International Conference on Power System Technology (POWERCON), 1-6, 2010.

[5] Chicco, Gianfranco, and Pierluigi Mancarella. "A Unified Model for Energy and Environmental Performance Assessment of Natural Gas-fueled Poly-generation Systems." Energy Conversion and Management 49, no. 8 (August 2008): 2069-2077.

[6] Manfroi, G., M. Maistrello, and L. C. Tagliabue. "Synergy of Geothermal Heat Pumps and PV Plant for Buildings Block.” In 2011 International Conf. on Clean Electrical Power (ICCEP), 466-473, 2011.

[7] Giraud, F., and Z.M. Salameh. "Steady-state Performance of a Gridconnected Rooftop Hybrid Wind-photovoltaic Power System with Battery Storage.” IEEE Trans. on Energy Conversion 16, no. 1 (March 2001): 1-7.

[8] Liu, Shujun, Zaijun Wu, Xiaobo Dou, Bo Zhao, Shanglin Zhao, and Chunjun Sun. "Optimal Configuration of Hybrid Solar-wind Distributed Generation Capacity in a Grid-connected Microgrid." In Innovative Smart Grid Technologies (ISGT), 2013 IEEE PES, 1-6, 2013.

[9] C. F. Calvillo, A. Sánchez-Miralles, and J. Villar. "Distributed Energy Generation in Smart Cities." In 2013 International Conference on Renewable Energy Research and Applications (ICRERA), unpublished.

[10] IDAE Secretaría General Departamento de Planificación y Estudios, "Análisis del consumo energético del sector residencial en España: INFORME FINAL”, July 2011.

[11] REE Red Eléctrica de España, "Proyecto INDEL: Atlas de la Demanda Eléctrica Española”, 1998. 
[12] EPA, US. "CHP Technologies." Accessed April 24, 2013. http://www.epa.gov/chp/technologies.html.

[13] NREL, "2010 Solar Technologies Market Report: November 2011". (2011). 121 pp. NREL Report.

[14] IPCC. "Special Report on Renewable Energy Sources and Climate Change Mitigation". Edited by O. Edenhofer, R. Pichs-Madruga, Y. Sokona, K. Seyboth, P. Matschoss, S. Kadner, T. Zwickel, et al. United Kingdom and New York, NY, USA: Cambridge University Press, 2011.

[15] NUS Consulting Group. "2011-012 International Electricity and Natural Gas, Report and Price Survey," June 2012.

[16] European Commission - Joint Research Centre, "Photovoltaic Geographical Information System - Interactive Maps.” Accessed April 24, 2013. http://re.jrc.ec.europa.eu/pvgis/apps4/pvest.php.

[17] IDAE, "Atlas Eólico de España". Accessed April 24, 2013. http://atlaseolico.idae.es/meteosim. 